\title{
ON F-DATA OF AUTOMORPHISM GROUPS OF COMPACT RIEMANN SURFACES
}

- THE CASE OF $A_{5}$

\author{
By HIDEYUKI KIMURA
}

\section{Introduction.}

Let $X$ be a compact Riemann surface of genus $g(\geqq 2)$. We denote by $\operatorname{Aut}(X)$ the group of all conformal automorphisms on $X$. We take a basis of the space of abelian differentials of the first kind on $X$. We consider the canonical representation $\rho: \operatorname{Aut}(X) \rightarrow G L(g, C)$, for the basis. We denote by $\rho(A G ; X)$ and $\rho(\sigma ; X)$ the images of a subgroup $A G \subset$ Aut $(X)$ and an element $\sigma \in \operatorname{Aut}(X)$ by $\rho$, respectively. In the previous paper [2], for the $G\left(\cong D_{8}, Q_{8}\right)$ $\subset G L(g, C)$, satisfying the $C Y$ - and $R H$-conditions, we have investigated surjective homomorphisms $\varphi: \Gamma(G) \rightarrow G$ to determine whether $G$ arises from a compact Riemann surface of genus $g$. But there exists $G\left(\cong A_{5}\right) \subset G L(g, C)$, satisfying the $C Y$ - and $R H$-conditions, such that we can not determine that $G$ arises from a compact Riemann surface of genus $g$ by the same method. Therefore we introduce the collection of nonnegative integers which consists of information about characters of $\rho$ and fixed points of $A G$. We shall call this $F$-data. In this paper, we study $F$-data of $A_{5}$ and determine what $F$-data of $A_{5}$ arises from a compact Riemann surface of genus $g$.

Notation. We denote by $\boldsymbol{Z}, \boldsymbol{C}$ and $\boldsymbol{Z}_{\geq 0}$ the ring of rational integers, the complex number field and the set of nonnegative integers, respectively. For a finite set $S$ we denote by $\# S$ the cardinality of $S$. For an element $\sigma$ of a finite group we denote by $\# \sigma$ the order of $\sigma$. We denote by $g$ an integer $(\geqq 2)$.

The author wishes to express his gratitude to the referees for their careful reading and valuable suggestions.

\section{$\S 1$. Preliminaries.}

In this section we give preliminary results. We use the same notation and terminology as introduced in [3]. Throughout this section we denote by $G$ a finite group.

\footnotetext{
Received January 11, 1990 ; revised February 27, 1991.
} 
Definition. We say that $G \subset G L(g, C)$ arises from a compact Riemann surface of genus $g$, if there exist a compact Riemann surface $X$ of genus $g$ and a subgroup $A G \subset \operatorname{Aut}(X)$ such that $\rho(A G ; X)$ is $G L(g, C)$-conjugate to $G$.

1.1 We give a necessary and sufficient condition for an element of prime order of $G L(g, C)$ to arise from a compact Riemann surface of genus $g$, see [4] and [7].

THEOREM ([4]). Let $A$ be an element of prime order $n$ of $G L(g, C)$. Then the following two conditions are equivalent:

(1) There is a compact Riemann surface $X$ of genus $g$ and an automorphism $\sigma$ of $X$ such that $\rho(\sigma ; X)$ is conjugate to $A$.

(2) There are $s(\geqq 0)$ integers $\nu_{1}, \cdots, \nu_{s}$ which are prime to $n$ such that $\operatorname{Tr} A=1+\sum_{i=1}^{s} \frac{\zeta^{\nu} i}{1-\zeta^{\nu} i}$, where $\zeta=\zeta_{n}=\exp \frac{2 \pi \sqrt{-1}}{n}$.

1.2 We define the $C Y$ - and $R H$-conditions, see [3] and [5].

Definition. We say that a finite group $G \subset G L(g, C)$ satisfies the $C Y$ condition if every element of $C Y(G)$ arises from a compact Riemann surface of genus $g$.

Remark. It is known that $A_{5}$ has only elements of prime orders, i.e., 2, 3 and 5. The above theorem, mentioned in 1.1 , suffices to check that $G\left(\cong A_{5}\right)$ satisfies the $C Y$-condition.

Definition. We say that $G$ satisfies the $R H$-condition if $G$ satisfies the $E$-condition and $l(H: G)$ is a nonnegative integer for any $H \in C Y(G)$.

1.3 Now, we introduce the $E X$-condition, which is a necessary condition for $G$ to arise from a compact Riemann surface. We explain a criterion whether $G$, satisfying the $E X$-condition, arises from a compact Riemann surface or not, see $[6]$.

Definition. Assume that $G \subset G L(g, C)$ satisfies the $R H$-condition. We say that $G$ satisfies the $E X$-condition if there exists a surjective homomorphism $\varphi: \Gamma(G) \rightarrow G$ with $\# \varphi\left(\gamma_{j}\right)=m_{j}(j=1, \cdots, r)$.

If $G \subset G L(g, C)$ satisfies the $E X$-condition, there exist a compact Riemann surface $X$ of genus $g$ and an injective homomorphism $G \rightarrow \operatorname{Aut}(X)$. Then for any element $\sigma(\# \sigma=m>1)$ of $G$ and $u \in \boldsymbol{Z}((u, m)=1)$ we have (cf. [6])

$$
\#\left\{P \in X \mid \zeta_{P}(\sigma)=\zeta_{m}^{u}\right\}=\sum_{m_{1} m_{j}} \frac{1}{m_{\jmath}} \#\left\{\alpha \in G \mid \sigma=\alpha \varphi\left(\gamma_{j}\right)^{u m_{j} / m} \alpha^{-1}\right\} .
$$

By the Eichler trace formula, we have 


$$
\operatorname{Tr} \rho(\sigma ; X)=1+\sum_{(u, m)=1} \sum_{m \mid m} \frac{1}{m_{\jmath}} \#\left\{\alpha \in G \mid \sigma=\alpha \varphi\left(\gamma_{j}\right)^{u m_{j} / m} \alpha^{-1}\right\} \frac{\zeta_{m}^{u}}{1-\zeta_{m}^{u}} .
$$

If there exists a surjective homomorphism $\varphi: \Gamma(G) \rightarrow G$ such

$$
\operatorname{Tr} \sigma=\operatorname{Tr} \rho(\sigma ; X) \quad \text { for every } \sigma \in G,
$$

then we see that $G$ arises from the compact Riemann surface $X$.

1.4 We denote by $A_{5}$ the alternating group of degree 5 , i.e., the group which consists of all the even permutations of 5 letters. The character table of $A_{5}$ is as follows:

\begin{tabular}{l|rrrrr} 
& $(1)$ & $(12)(34)$ & $(123)$ & $(12345)$ & $(13524)$ \\
\hline$\chi_{1}$ & 1 & 1 & 1 & 1 & 1 \\
$\chi_{2}$ & 4 & 0 & 1 & -1 & -1 \\
$\chi_{3}$ & 5 & 1 & -1 & 0 & 0 \\
$\chi_{4}$ & 3 & -1 & 0 & $\frac{1+\sqrt{5}}{2}$ & $\frac{1-\sqrt{5}}{2}$ \\
$\chi_{5}$ & & & & $1-\sqrt{5}$ & $\frac{1+\sqrt{5}}{2}$ \\
& & -1 & 0 & $\frac{1-1}{2}$
\end{tabular}

\section{§2. A necessary and sufficient condition for $C Y$ - and $R H$-conditions.}

2.1. Proposition. Let $G$ be a finite subgroup of $G L(g, C)$ being isomorphic to $A_{5}, \chi_{G}$ be the character of the natural representation $G \rightarrow G L(g, C)$. Let $n_{1} \chi_{1}+\cdots+n_{5} \chi_{5}, n_{i} \in Z_{\geq 0}$ be the decomposition into irreducible characters of $\chi_{G}$. Then $G$ satisfies the $C Y$ - and RH-conditions if and only if $n_{\imath}$ 's satisfy the following relations:
(1) $1 \geqq n_{1}+n_{3}-2 n_{4}$
(2) $1 \geqq n_{1}+n_{2}-n_{3}$
(3) $1 \geqq n_{1}-n_{2}+n_{4}$
(4) $n_{4}=n_{5}$.

Remark. If $G$ satisfies the $C Y$ - and $R H$-conditions, then we have

$$
\text { (0) } g=n_{1}+4 n_{2}+5 n_{3}+6 n_{4} \text {, }
$$

which means the degree of character $\chi_{G}$.

Proof. We prove the if-part. We fix an isomorphism $\iota: A_{5} \rightarrow G$ and denote 
by $A, B$ and $C$ the images of (23)(45), (142) and (12345) via $\iota$, respectively. We remark that, by Property 6 (I-1), two of $A, B$ and $C$ generate $G$. First we show that $G$ satisfies the $C Y$-condition. To see this, it is sufficient to show that $A, B$ and $C$ satisfy the condition (2) in Theorem 1.1.

The case of $A$.

Put

$$
s:=2-2 \chi_{G}(A)=2-2\left(n_{1}+n_{3}-n_{4}-n_{5}\right) .
$$

Then we see that $s$ is a nonnegative integer by (1) and (4). If we put

then we have

$$
\nu_{1}=\cdots=\nu_{s}=1
$$

$$
\operatorname{Tr} A=1+s \frac{-1}{1-(-1)} .
$$

Thus $A$ arises from a compact Riemann surface of genus $g$ by Theorem 1.1.

The case of $B$.

Put

$$
s:=2-2 \chi_{G}(B)=2-2\left(n_{1}+n_{2}-n_{3}\right) .
$$

Then we see that $s / 2$ is a nonnegative integer by (2). If we put

then we have

$$
\nu_{1}=\cdots=\nu_{s / 2}=1, \nu_{(s / 2)+1}=\cdots=\nu_{s}=2,
$$

$$
\operatorname{Tr} B=1+\frac{s}{2}\left(\frac{\omega}{1-\omega}+\frac{\omega^{2}}{1-\omega^{2}}\right), \text { where } \omega=\zeta_{3} .
$$

Thus $B$ arises from a compact Riemann surface of genus $g$ by Theorem 1.1 .

The case of $C$.

Put

$$
s:=2-2 \chi_{G}(C)=2-2\left(n_{1}-n_{2}+\frac{1+\sqrt{5}}{2} n_{4}+\frac{1-\sqrt{5}}{2} n_{5}\right) .
$$

Then we see that $s / 2$ is a nonnegative integer by (3) and (4). We take $p, q \in Z_{\geqq 0}$ with $p+q=s / 2$. If we put

then we have

$$
\begin{aligned}
& \nu_{1}=\cdots=\nu_{p}=1, \nu_{p+1}=\cdots=\nu_{2 p}=4, \\
& \nu_{2 p+1}=\cdots=\nu_{2 p+q}=2, \nu_{2 p+q+1}=\cdots=\nu_{s}=3,
\end{aligned}
$$

$$
\operatorname{Tr} C=1+p\left(\frac{\zeta}{1-\zeta}+\frac{\zeta^{4}}{1-\zeta^{4}}\right)+q\left(\frac{\zeta^{2}}{1-\zeta^{2}}+\frac{\zeta^{3}}{1-\zeta^{3}}\right),
$$

where $\zeta=\zeta_{5}$. 
Thus $C$ arises from a compact Riemann surface of genus $g$ by Theorem 1.1.

This means that $G$ satisfies the $C Y$-condition. It is easy to see that $G$ satisfies the $R H$-condition. In fact we have

$$
\begin{aligned}
& l(\langle A\rangle: G)=1-\left(n_{1}+n_{3}-n_{4}-n_{5}\right) \\
& l(\langle B\rangle: G)=1-\left(n_{1}+n_{2}-n_{3}\right) \\
& l(\langle C\rangle: G)=1-\left(n_{1}-n_{2}+\frac{1+\sqrt{5}}{2} n_{4}+\frac{1-\sqrt{5}}{2} n_{5}\right),
\end{aligned}
$$

which are nonnegative integers by (1), $\cdots,(4)$.

The only-if-part follows immediately from the fact:

$$
\chi_{G}(C)=\chi_{G}\left(C^{2}\right) \text { implies } n_{4}=n_{5} .
$$

Therefore we obtain our proposition.

Remark. In the case of $B$, since $B$ is $G$-conjugate to $B^{2}$, we have

$$
\#\left\{i \mid \nu_{i}=1\right\}=\#\left\{i \mid \nu_{i}=2\right\} \text {. }
$$

In the case of $C$, since $C$ (resp. $\left.C^{2}\right)$ is $G$-conjugate to $C^{4}\left(\right.$ resp. $\left.C^{3}\right)$, we have

$$
\left.\#\left\{i \mid \nu_{i}=1\right\}=\#\left\{i \mid \nu_{i}=4\right\} \quad \text { (resp. } \#\left\{i \mid \nu_{i}=2\right\}=\#\left\{i \mid \nu_{i}=3\right\}\right) .
$$

2.2 We introduce an $F$-data of $A_{5}$.

Definition. We say that a collection of nonnegative integers $\left(n_{1}, \cdots, n_{5}\right.$; $p, q), p \geqq q$, is an $F$-data of $A_{5}$ if there exists a group $G\left(\cong A_{5}\right) \subset G L(g, C)$ satisfying the $C Y$ - and $R H$-conditions and that

$$
\begin{aligned}
& \chi_{G}=n_{1} \chi_{1}+\cdots+n_{5} \chi_{5}, \\
& 1-\chi_{G}(C)=p+q \quad \text { for every } \quad C(\in G) \text { of order } 5 .
\end{aligned}
$$

Instead of $G\left(\cong A_{5}\right) \subset G L(g, C)$ which satisfies the $C Y$ - and $R H$-conditions, we consider an $F$-data $\left(n_{1}, \cdots, n_{5} ; p, q\right)$ of $A_{5}$.

Definition. Let $\left(n_{1}, \cdots, n_{5} ; p, q\right)$ be an $F$-data of $A_{5}$. Define $g$ by $(0)$. We say that an $F$-data $\left(n_{1}, \cdots, n_{5} ; p, q\right)$ of $A_{5}$ arises from a compact Riemann surface of genus $g$ if there exist a compact Riemann surface $X$ of genus $g$, a subgroup $A G\left(\cong A_{5}\right) \subset \operatorname{Aut}(X)$ and an element $C(\in A G)$ of order 5 such that

and

$$
\left.\operatorname{Tr} \rho(\circ ; X)\right|_{A G}=n_{1} \chi_{1}+\cdots+n_{5} \chi_{5}=\chi_{G}
$$

$$
\left.\begin{array}{l}
\#\left\{P \in X \mid \zeta_{P}(C)=\zeta\right\}=\#\left\{P \in X \mid \zeta_{P}(C)=\zeta^{4}\right\}=p, \\
\#\left\{P \in X \mid \zeta_{P}(C)=\zeta^{2}\right\}=\#\left\{P \in X \mid \zeta_{P}(C)=\zeta^{3}\right\}=q .
\end{array}\right\}
$$




\section{§3. Characterization of automorphism groups.}

Hereafter, for simplicity, we put $l(\circ)=l(\circ: G)=l(\langle\circ\rangle: G)$.

3.1. Theorem. The notation being as in Proposition 2.1 , let $\left(n_{1}, \cdots, n_{5} ; p, q\right)$ be an $F$-data of $A_{5}$. If $\left(n_{1}, \cdots, n_{5} ; p, q\right)$ does not arise from a compact Riemann surface of genus $g$, then

or

$$
\begin{aligned}
\left(n_{1}, \cdots, n_{5} ; p, q\right) & =(0,1,0,0,0 ; 2,0) \quad(g=4), \\
& =(0,2,1,0,0 ; 2,1) \quad(g=13)
\end{aligned}
$$$$
=(1,1,1,1,1 ; 0,0) \quad(g=16) .
$$

Remark. The $F$-data $(0,1,0,0,0 ; 1,1)$ (resp. $(0,2,1,0,0 ; 3,0))$ arises from a compact Riemann surface of genus 4 (resp. 13) but not $(0,1,0,0,0 ; 2,0)$ (resp. $(0,2,1,0,0 ; 2,1)$ ).

Before proving the theorem, we give some properties of $A_{5}$.

Property 1.

For every element $h$ of $A_{5}$, there exist elements $a, b\left(\in A_{5}\right)$ such that $h=$ $[a, b]$, where $[a, b]=a b a^{-1} b^{-1}$.

Proof. It is sufficient to consider representatives of conjugacy classes of $A_{5}$, since we have the relation:

for $g \in A_{5}$.

$$
g^{-1}[x, y] g=\left[g^{-1} x g, g^{-1} y g\right]
$$

(1) order 2.

Put

Then we have

$$
a=(234), \quad b=(134)
$$

(2) order 3.

$[a, b]=(12)(34)$.

Put

Then we have

$$
a=(13)(45), \quad b=(23)(35) \text {. }
$$

(3) order 5.

Put

$$
[a, b]=(123) \text {. }
$$

Then we have

$$
\begin{array}{ll}
a_{1}=(25)(34), & b_{1}=(13)(45), \\
a_{2}=(25)(34), & b_{2}=(15)(24) .
\end{array}
$$

Property 2.

$$
\left[a_{1}, b_{1}\right]=(12345), \quad\left[a_{2}, b_{2}\right]=(13524) .
$$


For every $\theta \in A_{5}, \theta$ is $A_{5}$-conjugate to $\theta^{-1}$.

Proof. In the case $\# \theta=2,3$, it is easily verified from the character table of $A_{5}$. In the case $\# \theta=5$, it is verified from the following relation:

$$
(12345)=(25)(34)(12345)^{-1}(25)(34) \text {. }
$$

Property 3.

Let $\varepsilon$ and $\varepsilon^{\prime}$ be elements of $A_{5}$ of order 5 . If $\varepsilon$ is $A_{5}$-conjugate to $\varepsilon^{\prime}$, then'the order of $\varepsilon \varepsilon^{\prime}$ is not 2 .

Property 4.

Let $\varepsilon$ and $\varepsilon^{\prime}$ be distinct elements of $A_{5}$ of order 5 . If $\varepsilon$ is $A_{5}$-conjugate to $\varepsilon^{\prime}$ and $\varepsilon \varepsilon^{\prime}$ is of order 5 , then $\varepsilon \varepsilon^{\prime}$ is $A_{5}$-conjugate to $\varepsilon$.

To prove Properties 3 and 4, we use a result from character theory (c.f. $[\mathrm{G}])$ :

Theorem. Denote the conjugacy classes of finite group $G$ by $K_{2}$ and let $y_{2}$ be an element of $K_{\imath}, 1 \leqq i \leqq r$. Then if $\lambda_{\imath \jmath k}$ is the number of times of given element of $K_{k}$ can be expressed as an ordered product of an element of $K_{\imath}$ and an element of $K_{\jmath}$, we have

$$
\lambda_{\imath j k}=\frac{\# K_{i} \cdot \# K_{j}}{\# G} \sum_{m=1}^{r} \frac{\chi_{m}\left(y_{i}\right) \chi_{m}\left(y_{j}\right) \overline{\chi_{m}\left(y_{k}\right)}}{\chi_{m}(1)}
$$

for $1 \leqq i, \jmath, k \leqq r$.

Proof of Property 3. We apply the above theorem. We take a conjugacy class of order 5 as $K_{\imath}$ and the conjugacy class of order 2 as $K_{k}$. Put $K_{\jmath}=K_{\imath}$. Then we have $\lambda_{\imath j k}=0$. This means that there are no elements $\varepsilon, \varepsilon^{\prime} \in K_{\imath}$ such that $\varepsilon \varepsilon^{\prime} \in K_{k}$.

Proof of Property 4. We take a conjugacy class of order 5 as $K_{2}$ and the other conjugacy class of order 5 as $K_{k}$. Put $K_{\jmath}=K_{\imath}$. Then we have $\lambda_{\imath j_{k}}=1$. This means than $x=y=z^{3}$ is the unique solution of the equation $z=x \cdot y\left(z \in K_{k}\right.$, $x, y \in K_{\imath}$ ). This completes the proof.

\section{Property 5.}

Let $\theta$ be an element of $A_{5}$ and $N$ a positive integer. Then there are $N$ elements $\theta_{1}, \cdots, \theta_{N}\left(\in A_{5}\right.$, not necessarily distinct) being $A_{5}$-conjugate to $\theta$ such that $\theta=\theta_{1} \cdots \theta_{N}$.

Proof. This follows from the relations $(12)(34)=(13)(24) \cdot(14)(23), \quad(14352)=$ $(12345) \cdot(13425),(14325)=(13524) \cdot(12354)$. We prove only the case $\# \theta=2$. Case $N \equiv 0(\bmod 2)$. The above relation means that there exist elements $\theta^{\prime}, \theta^{\prime \prime}$ of order 2 such that $\theta=\theta^{\prime} \times \theta^{\prime \prime}$. Then we may take $\theta_{1}=\theta^{\prime}, \theta_{2}=\cdots=\theta_{N}=\theta^{\prime \prime}$. Case $N \equiv 1(\bmod 2)$. We may take $\theta_{1}=\cdots=\theta_{N}=\theta$. 
Property 6.

We have the following presentations ( I -1$), \cdots,(\mathrm{VI}-1)$ for $A_{5}$.

( I -1) $A_{5}=\left\langle\gamma, \delta, \varepsilon ; \gamma^{2}=\delta^{3}=\varepsilon^{5}=\gamma \delta \varepsilon=1\right\rangle$.

(for example, $\gamma=(23)(45), \delta=(142), \varepsilon=(12345)$ ).

In the following, we write only relations and mean that $\varepsilon_{2}$ 's are $A_{5}$-conjugate to each other and $\varepsilon_{\imath}$ 's are not $A_{5}$-conjugate to $\eta_{i}$ 's.

( I -2) $\left(\gamma_{1}\right)^{2}=\left(\gamma_{2}\right)^{2}=\left(\gamma_{3}\right)^{2}=\varepsilon^{5}=\gamma_{1} \gamma_{2} \gamma_{3} \varepsilon=1$,

(for example, $\gamma_{1}=(23)(45), \gamma_{2}=(12)(35), \gamma_{3}=(14)(35), \varepsilon=(12345)$ ).

( I -3) $\gamma^{2}=\left(\delta_{1}\right)^{3}=\left(\delta_{2}\right)^{3}=\left(\delta_{3}\right)^{3}=\gamma \delta_{1} \delta_{2} \delta_{3}=1$,

(for example, $\gamma=(23)(45), \delta_{1}=(142), \delta_{2}=(123), \delta_{3}=(345)$ ).

( I -4) $\gamma^{2}=\left(\varepsilon_{1}\right)^{5}=\left(\varepsilon_{2}\right)^{5}=\left(\varepsilon_{3}\right)^{5}=\gamma \varepsilon_{1} \varepsilon_{2} \varepsilon_{3}=1$,

(for example, $\gamma=(23)(45), \varepsilon_{1}=\varepsilon_{3}=(12345), \varepsilon_{2}=(13254)$ ).

( I -5) $\left(\gamma_{1}\right)^{2}=\left(\gamma_{2}\right)^{2}=\left(\gamma_{3}\right)^{2}=\left(\gamma_{4}\right)^{2}=\left(\gamma_{5}\right)^{2}=\gamma_{1} \gamma_{2} \gamma_{3} \gamma_{4} \gamma_{5}=1$,

(for example, $\gamma_{1}=(23)(45), \gamma_{2}=(12)(35), \gamma_{3}=(14)(35), \gamma_{4}=(15)(24), \gamma_{5}=(14)(23)$ ).

( I -6) $\left(\gamma_{1}\right)^{2}=\left(\gamma_{2}\right)^{2}=\left(\gamma_{3}\right)^{2}=\delta^{3}=\gamma_{1} \gamma_{2} \gamma_{3} \delta=1$,

(for example, $\gamma_{1}=(15)(24), \gamma_{2}=(14)(23), \gamma_{3}=(23)(45), \delta=(142)$ ).

( I -7) $\left(\gamma_{1}\right)^{2}=\left(\gamma_{2}\right)^{2}=\left(\varepsilon_{1}\right)^{5}=\left(\varepsilon_{2}\right)^{5}=\gamma_{1} \gamma_{2} \varepsilon_{1} \varepsilon_{2}=1$,

(for example, $\gamma_{1}=\gamma_{2}=(23)(45), \varepsilon_{1}=(15432), \varepsilon_{2}=(12345)$ ).

( I -8) $\left(\gamma_{1}\right)^{2}=\left(\gamma_{2}\right)^{2}=\left(\gamma_{3}\right)^{2}=\varepsilon^{5}=\eta^{5}=\gamma_{1} \gamma_{2} \gamma_{3} \varepsilon \eta=1$,

(for example, $\gamma_{1}=\gamma_{3}=(14)(35), \gamma_{2}=(12)(35), \varepsilon=(12345), \quad \eta=(15234)$ ).

( I -9) $\gamma^{2}=\delta^{3}=\left(\varepsilon_{1}\right)^{5}=\left(\varepsilon_{2}\right)^{5}=\gamma \delta \varepsilon_{1} \varepsilon_{2}=1$,

(for example, $\gamma=(23)(45), \delta=(142), \varepsilon_{1}=(14352), \varepsilon_{2}=(15243)$ ).

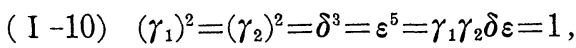

(for example, $\gamma_{1}=(24)(35), \gamma_{2}=(25)(34), \delta=(142), \varepsilon=(12345)$ ).

( I -11) $\gamma^{2}=\left(\delta_{1}\right)^{3}=\left(\delta_{2}\right)^{3}=\varepsilon^{5}=\gamma \delta_{1} \delta_{2} \varepsilon=1$,

(for example, $\gamma=(23)(45), \delta_{1}=\delta_{2}=(124), \varepsilon=(12345)$ ).

(II -1) $\gamma^{2}=\varepsilon^{5}=\eta^{5}=\gamma \varepsilon \eta=1$,

(for example, $\gamma=(13)(25), \varepsilon=(12345), \eta=(12435)$ ).

(II -2) $\left(\gamma_{1}\right)^{2}=\left(\gamma_{2}\right)^{2}=\varepsilon^{5}=\eta^{5}=\gamma_{1} \gamma_{2} \varepsilon \eta=1$,

(for example, $\gamma_{1}=(12)(35), \gamma_{2}=(15)(23), \varepsilon=(12345), \eta=(12435)$ ).

(II -3) $\gamma^{2}=\delta^{3}=\varepsilon^{5}=\eta^{5}=\gamma \delta \varepsilon \eta=1$,

(for example, $\gamma=(13)(45), \delta=(245), \varepsilon=(12345), \eta=(12435)$ ). 
(III-1) $\left(\delta_{1}\right)^{3}=\left(\delta_{2}\right)^{3}=\varepsilon^{5}=\delta_{1} \delta_{2} \varepsilon=1$,

(for example, $\delta_{1}=(354), \delta_{2}=(132), \varepsilon=(12345)$ ).

(III-2) $\left(\gamma_{1}\right)^{2}=\left(\gamma_{2}\right)^{2}=\left(\delta_{1}\right)^{3}=\left(\delta_{2}\right)^{3}=\gamma_{1} \gamma_{2} \delta_{1} \delta_{2}=1$,

(for example, $\gamma_{1}=(15)(24), \gamma_{2}=(14)(23), \delta_{1}=(354), \delta_{2}=(132)$ ).

(III-3) $\left(\delta_{1}\right)^{3}=\left(\delta_{2}\right)^{3}=\left(\delta_{3}\right)^{3}=\left(\delta_{4}\right)^{3}=\delta_{1} \delta_{2} \delta_{3} \delta_{4}=1$,

(for example, $\delta_{1}=(354), \delta_{2}=(132), \delta_{3}=(123), \delta_{4}=(345)$ ).

$(\mathrm{IV}-1) \quad \delta^{3}=\left(\varepsilon_{1}\right)^{5}=\left(\varepsilon_{2}\right)^{5}=\delta \varepsilon_{1} \varepsilon_{2}=1$,

(for example, $\delta=(134), \varepsilon_{1}=(12345), \varepsilon_{2}=(13542)$ ).

$(\mathrm{V}-1) \quad \delta^{3}=\varepsilon^{5}=\eta^{5}=\delta \varepsilon \eta=1$,

(for example, $\delta=(145), \varepsilon=(12345), \eta=(14532)$ ).

$(\mathrm{V}-2) \quad\left(\varepsilon_{1}\right)^{5}=\left(\varepsilon_{2}\right)^{5}=\left(\varepsilon_{3}\right)^{5}=\eta^{5}=\varepsilon_{1} \varepsilon_{2} \varepsilon_{3} \eta=1$,

(for example, $\varepsilon_{1}=(13542), \varepsilon_{2}=(15243), \varepsilon_{3}=(12345), \eta=(14532)$ ).

$(\mathrm{V}-3) \quad\left(\delta_{1}\right)^{3}=\left(\delta_{2}\right)^{3}=\varepsilon^{5}=\eta^{5}=\delta_{1} \delta_{2} \varepsilon \eta=1$,

(for example, $\delta_{1}=\delta_{2}=(154), \varepsilon=(12345), \eta=(14532)$ ).

$(\mathrm{V}-4) \quad \varepsilon^{5}=\eta^{5}=\left(\varepsilon \varepsilon^{-1} \eta \eta^{-1}\right)=1$,

(for example, $\varepsilon=(15432), \eta=(14532)$ ).

(VI-1) $\left(\varepsilon_{1}\right)^{5}=\left(\varepsilon_{2}\right)^{5}=\left(\varepsilon_{3}\right)^{5}=\varepsilon_{1} \varepsilon_{2} \varepsilon_{3}=1$,

(for example, $\varepsilon_{1}=(12345), \varepsilon_{2}=(12534), \varepsilon_{3}=(12453)$ ).

Proof of Property 6. It is sufficient to verify that $\gamma, \delta, \varepsilon, \cdots$ generate $A_{5}$. Here we remark the following FACT.

FACT. (c.f. [ATLAS]) Maximal subgroups of $A_{5}$ are $A_{4}, D_{10}$ and $S_{3}$. For example, we consider the case (I-1). If $\langle\gamma, \delta, \varepsilon\rangle$ is a proper subgroup of $A_{5}$, since it has an element of order $5, D_{10} \supset\langle\gamma, \delta, \varepsilon\rangle$. However, $D_{10}$ does not contain an element of order 3. This means $A_{5}=\langle\gamma, \delta, \varepsilon\rangle$. The remaining cases are proved by the similar method.

Proof of Theorem. Let the notation be as in Proposition 2.1. Recall that

and put

$$
R H(G)=\left[n_{1}, 60 ; 2, \cdots, 2,3, \cdots, 3,5, \cdots, 5\right]
$$

$$
\begin{aligned}
& \Gamma(G)=\left\langle\alpha_{1}, \beta_{1}, \cdots, \alpha_{n_{1}}, \beta_{n_{1}}, \gamma_{1}, \cdots, \gamma_{l(A)}, \delta_{1}, \cdots, \delta_{l(B)}, \varepsilon_{1}, \cdots, \varepsilon_{p}, \eta_{1}, \cdots, \eta_{q} ;\right. \\
& \prod_{\jmath=1}^{l(A)} \gamma_{j} \prod_{k=1}^{l(B)} \delta_{k} \prod_{l=1}^{p} \varepsilon_{l} \prod_{m=1}^{q} \eta_{m} \prod_{\imath=1}^{n_{1}}\left[\alpha_{\imath}, \beta_{l}\right]=1 \\
& \left.\gamma_{1}^{2}=\cdots=\gamma_{l(A)}^{2}=\delta_{1}^{3}=\cdots=\delta_{l(B)}^{3}=\varepsilon_{1}^{5}=\cdots=\varepsilon_{p}^{5}=\eta_{1}^{5}=\cdots=\eta_{q}^{5}=1\right\rangle .
\end{aligned}
$$


We study whether $G$ in Definition 2.2 satisfies the $E X$-condition or not. Using $\varphi$ 's, we verify whether $\left(n_{1}, \cdots, n_{5} ; p, q\right)$ satisfy condition $(*)$. We divide our proof into three cases according as $n_{1} \geqq 2, n_{1}=1$ or $n_{1}=0$.

We assume that $n_{1} \geqq 2$. We define $\varphi: \Gamma(G) \rightarrow G$ as follows: $\gamma_{1}, \cdots, \gamma_{l(A)} \rightarrow$ $A, \delta_{1}, \cdots, \delta_{l(B)} \rightarrow B, \varepsilon_{1}, \cdots, \varepsilon_{p} \rightarrow C, \eta_{1}, \cdots, \eta_{q} \rightarrow C^{2}, \alpha_{1} \rightarrow A, \beta_{1} \rightarrow B, \alpha_{2} \rightarrow U, \beta_{2} \rightarrow$ $V, \alpha_{3}, \beta_{3}, \cdots, \alpha_{n_{1}}, \beta_{n_{1}} \rightarrow 1$, where we choose $U$ and $V$ so that $\varphi\left(\Pi \gamma_{j} \Pi \delta_{k} \Pi \varepsilon_{l} \Pi \eta_{m}\right.$ $\left.\Pi\left[\alpha_{2}, \beta_{i}\right]\right)=1$ holds. By virtue of Property 1 , we can find them. Recall that $A$ and $B$ are the images of (23)(45) and (142), respectively. By Property 6 ( $I-1)$ we see that $\varphi$ is surjective. To verify $\operatorname{Tr} \rho(\circ ; X)=\chi_{G}(\circ)$, it is sufficient to check only for $\sigma=A, B$ and $C$.

$$
\begin{aligned}
& \operatorname{Tr} \rho(A ; X)=1+2 l(A) \frac{-1}{1+1}=1-l(A)=\chi_{G}(A) . \\
& \operatorname{Tr} \rho(B ; X)=1+l(B)\left(\frac{\omega}{1-\omega}+\frac{\omega^{2}}{1-\omega^{2}}\right)=1-l(B)=\chi_{G}(B),
\end{aligned}
$$

where $\omega=\zeta_{3}$.

$$
\begin{aligned}
\operatorname{Tr} \rho(C ; X) & =1+p\left(\frac{\zeta}{1-\zeta}+\frac{\zeta^{4}}{1-\zeta^{4}}\right)+q\left(\frac{\zeta^{2}}{1-\zeta^{2}}+\frac{\zeta^{3}}{1-\zeta^{3}}\right) \\
& =1-l(C)=\chi_{G}(C),
\end{aligned}
$$

where $\zeta=\zeta_{5}$.

Thus, in this case, we see that $\left(n_{1}, \cdots, n_{5} ; p, q\right)$ arises from a compact Riemann surface of genus $g$. In the following cases, we define only those $\varphi$ 's which have the desired property.

Next, we assume that $n_{1}=1$.

(i) The case $l(A)>0 \& l(B)>0$.

We define $\varphi: \Gamma(G) \rightarrow G$ as follows : $\gamma_{1}, \cdots, \gamma_{l(A)} \rightarrow A, \delta_{1}, \cdots, \delta_{l(B)} \rightarrow B, \varepsilon_{1}, \cdots, \varepsilon_{p} \rightarrow$ $C, \eta_{1}, \cdots, \eta_{q} \rightarrow C^{2}, \alpha_{1} \rightarrow U, \beta_{1} \rightarrow V$, where we choose $U$ and $V$ so that $\varphi\left(\Pi \gamma_{j} \Pi \delta_{k} \Pi \varepsilon_{l} \Pi \Pi \eta_{m} \Pi\left[\alpha_{\imath}, \beta_{\imath}\right]\right)=1$ holds. By virtue of Property 1, we can find them.

(ii) The case $l(A)=l(B)=0$.

By the assumption, we have $l(C)=n_{4} \geqq 1$.

(ii-1) $l(C)=n_{4}=1$.

Recall that we fix isomorphism $\iota: A_{5} \rightarrow G$. We define $\varphi: \Gamma(G) \rightarrow G$ as follows: $\varepsilon_{1} \rightarrow \iota((12453)), \quad \alpha_{1} \rightarrow \iota((354)), \quad \beta_{1} \rightarrow \iota((13)(25))$.

(ii-2) $l(C)=n_{4} \geqq 2$.

(ii-2-a) $\quad p \geqq 2$.

We define $\varphi: \Gamma(G) \rightarrow G$ as follows : $\varepsilon_{1} \rightarrow \iota((12345)), \varepsilon_{2}, \cdots, \varepsilon_{p} \rightarrow \iota((12534)), \eta_{1}, \cdots, \eta_{q}$ $\rightarrow \iota\left((12534)^{2}\right), \quad \alpha_{1} \rightarrow U, \beta_{1} \rightarrow V$, where we choose $U$ and $V$ so that $\varphi\left(\Pi \gamma_{j} \Pi \delta_{k} \Pi \varepsilon_{l}\right.$ $\left.\Pi \eta_{m} \Pi\left[\alpha_{\imath}, \beta_{\imath}\right]\right)=1$ holds. 
(ii-2-b) $\quad p=q=1$.

We define $\varphi: \Gamma(G) \rightarrow G$ as follows : $\varepsilon_{1} \rightarrow \iota((12345)), \quad \eta_{1} \rightarrow \iota((12435)), \quad \alpha_{1} \rightarrow U, \quad \beta_{1} \rightarrow V$, where we choose $U$ and $V$ so that $\varphi\left(\Pi \gamma_{j} \Pi \delta_{k} \Pi I \varepsilon_{l} \Pi \eta_{m} \Pi\left[\alpha_{\imath}, \beta_{\imath}\right]\right)=1$ holds.

(iii) The case $l(A)=0 \& l(B)>0$.

(iii-1) $l(C)>0$.

We define $\varphi: \Gamma(G) \rightarrow G$ as follows: $\delta_{1}, \cdots, \delta_{l(B)} \rightarrow B, \varepsilon_{1}, \cdots, \varepsilon_{p} \rightarrow C, \eta_{1}, \cdots, \eta_{q} \rightarrow$ $C^{2}, \alpha_{1} \rightarrow U, \beta_{1} \rightarrow V$, where we choose $U$ and $V$ so that $\varphi\left(\Pi \gamma_{j} \Pi \delta_{k} \Pi \varepsilon_{l} \Pi \eta_{m} \Pi\left[\alpha_{\imath}, \beta_{\imath}\right]\right)$ $=1$ holds.

(iii-2) $l(C)=0$.

By the assumption, we have $l(B)=n_{4} \geqq 1$.

Considering Property 5, we can reduce this case to $\delta_{1} \rightarrow \iota((134)), \alpha_{1} \rightarrow \iota((12345))$, $\beta_{1} \rightarrow \iota((12435))$.

(iv) The case $l(A)>0 \& l(B)=0$.

(iv-1) $l(C)>0$.

We define $\varphi: \Gamma(G) \rightarrow G$ as follows: $\gamma_{1}, \cdots, \gamma_{l(A)} \rightarrow A, \varepsilon_{1}, \cdots, \varepsilon_{p} \rightarrow C, \eta_{1}, \cdots, \eta_{q} \rightarrow$ $C^{2}, \alpha_{1} \rightarrow U, \beta_{1} \rightarrow V$, where we choose $U$ and $V$ so that $\varphi\left(\Pi \gamma_{j} \Pi \delta_{k} \Pi \varepsilon_{\imath} \Pi \Pi \eta_{m} \Pi\left[\alpha_{\imath}, \beta_{\imath}\right]\right)$ $=1$ holds.

(iv-2) $l(C)=0$.

(iv-2-a) $l(A) \geqq 2$.

Considering Property 4 , we can reduce this case to $\gamma_{1} \rightarrow \iota((25)(34)), \gamma_{2} \rightarrow \iota((14)(25))$, $\alpha_{1} \rightarrow \iota((12345)), \beta_{1} \rightarrow \iota((12435))$.

(iv-2-b) $l(A)=1$. (i.e. $n_{1}=n_{2}=n_{3}=n_{4}=n_{5}=1, p=q=0$.)

In this case, there is no $\varphi$ having the desired properties. To see this, it is sufficient to show that there are no elements $\alpha, \beta\left(\in A_{5}\right)$ such that $A_{5}=\langle\alpha, \beta|$ $\#[\alpha, \beta]=2>$.

The case $\# \alpha=5$.

Since $\alpha$ is $A_{5}$-conjugate to $\beta \alpha^{-1} \beta^{-1}$, by Property $3, \#[\alpha, \beta] \neq 2$.

The case $\# \alpha=\# \beta=3$.

By the abstract definition of $A_{4}$, i.e., $A_{4}=\left\langle S, T \mid S^{3}=T^{3}=(S T)^{2}=1\right\rangle$, we have $\#(\alpha \beta) \neq 2, \#\left(\alpha^{-1} \beta\right) \neq 2$, (of course $\#(\alpha \beta) \neq 1, \#\left(\alpha^{-1} \beta\right) \neq 1$ ). Suppose that $\#(\alpha \beta)=$ $\#\left(\alpha^{-1} \beta\right)=3$. Then $\langle\alpha, \beta\rangle$ must be contained in $(3,3 \mid 3,3)$ which is a group of order 27 , see [1]. This is absurd.

Therefore we have $\#(\alpha \beta)=5$ or $\#\left(\alpha^{-1} \beta\right)=5$. Assume $\#(\alpha \beta)=5$. Since $\alpha \beta$ is $A_{5}$-conjugate to $\alpha^{-1} \beta^{-1}$, by Property $3, \#[\alpha, \beta] \neq 2$. Next assume $\#\left(\alpha^{-1} \beta\right)=5$. Since $\alpha^{-1} \beta$ is $A_{5}$-conjugate to $\alpha \beta^{-1}$, by Property $3, \#\left[\alpha^{-1}, \beta\right]=\#\left(\alpha^{-1}[\alpha, \beta] \alpha\right)=$ $\#[\alpha, \beta] \neq 2$.

The case $\# \alpha=3 \& \# \beta=2$.

By the abstract definitions of $A_{4}$ and $S_{3}$, i.e., $A_{4}=\left\langle S, T \mid S^{3}=T^{2}=(S T)^{3}=1\right\rangle$,

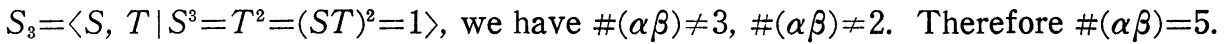


Since $\alpha \beta$ is $A_{5}$-conjugate to $\alpha^{-1} \beta^{-1}$, by Property $3, \#[\alpha, \beta] \neq 2$.

The case $\# \alpha=\# \beta=2$.

Assume $\#[\alpha, \beta]=2$. Then we have

$$
[\alpha, \beta]^{2}=1 \longleftrightarrow \alpha \beta=\beta \alpha \longleftrightarrow[\alpha, \beta]=1 .
$$

This is contradiction.

Thus we see that the $F$-data $(1,1,1,1,1 ; 0,0)$ does not arise from a compact Riemann surface of genus 16 .

Finally, we assume that $n_{1}=0$. Then

$$
l(A)=1-n_{3}+2 n_{4}, \quad l(B)=1-n_{2}+n_{3}, \quad l(C)=1+n_{2}-n_{4} .
$$

By simple calculation we see that the triple $(l(A), l(B), l(C))$ does not coincide any one of following:

$$
\begin{aligned}
& (0,0,0),(1,0,0),(0,1,0),(2,0,0),(1,1,0),(0,2,0),(3,0,0), \\
& (2,1,0),(1,2,0),(0,3,0),(4,0,0), \\
& (0,0,1),(1,0,1),(0,1,1),(2,0,1), \\
& (0,0,2),(1,1,1) .
\end{aligned}
$$

In the following, instead of defining $\varphi$, we give the relation (of $A_{5}$ ) which guarantees the extistence of $\varphi$.

(i) The case $l(A)=0 \quad \& \quad l(B)=0 \quad \& \quad l(C) \geqq 3$.

(i-1) $p \geqq 3, q=0$.

(i-2) $p \geqq 3, q=1$.

(i-3) $p \geqq 2, q \geqq 2$,

(i-4) $\quad p=2, q=1$.

Considering Property 5, we can reduce (i-1), (i-2) and (i-3) to Property 6 (VI-1), $(\mathrm{V}-2)$ and $(\mathrm{V}-4)$, respectively. In the case of $(\mathrm{i}-4)$, by Property 4 , the $F$-data $(0,2,1,0,0 ; 2,1)$ does not arise from a compact Riemann surface of genus 13 .

(ii) The case $l(A) \geqq 1 \& l(B) \geqq 1 \& l(C) \geqq 2$.

(ii-1) $\quad p \geqq 2, q=0$.

(ii-2) $\quad p \geqq 1, q \geqq 1$.

Considering Property 5, we can reduce (ii-1) and (ii-2) to Property 6 (I-9) and (II -3), respectively.

(iii) The case $l(A) \geqq 2 \& l(B) \geqq 1 \quad \& \quad l(C)=1$.

Considering Property 5, we can reduce this case to Property 6 (I-10).

(iv) The case $l(A)=1 \& l(B) \geqq 2 \& l(C)=1$.

Considering Property 5, we can reduce this case to Property 6 (I-11). 
(v) The case $l(A) \geqq 3 \quad \& l(B)=0 \quad \& \quad l(C) \geqq 1$.

(v-1) $\quad p \geqq 1, q=0$.

$(\mathrm{v}-2) \quad p \geqq 1, q \geqq 1$.

Considering Property 5, we can reduce ( $\mathrm{v}-1)$ and (v-2) to Property 6 (I-2) and (I-8), respectively.

(vi) The case $l(A)=0 \quad \& \quad l(B) \geqq 2 \quad \& \quad l(C) \geqq 1$.

(vi-1) $\quad p \geqq 1, q=0$.

(vi-2) $\quad p \geqq 1, q \geqq 1$.

Considering Property 5, we can reduce (vi-1) and (vi-2) to Property 6 (III-1) and (V-3), respectively.

(vii) The case $l(A)=1 \quad \& \quad l(B)=0 \quad \& \quad l(C) \geqq 2$.

(vii-1) $p \geqq 3, q=0$.

(vii-2) $p \geqq 1, q \geqq 1$.

(vii-3) $\quad p=2, q=0$.

Considering Property 5, we can reduce (vii-1) and (vii-2) to Property 6 (I-4) and (II-1), respectively. In the case of (vii-3), by Property 3 , the $F$-data $(0,1,0,0$, $0 ; 2,0)$ does not arise from a compact Riemann surface of genus 4 .

(viii) The case $l(A)=2 \& l(B)=0 \quad \& \quad l(C) \geqq 2$.

(viii-1) $\quad p \geqq 2, q=0$.

(viii-2) $\quad p \geqq 1, q \geqq 1$.

Considering Property 5, we can reduce (viii-1) and (viii-2) to Property 6 (I-7) and (II-2), respectively.

(ix) The case $l(A)=0 \quad \& \quad l(B)=1 \quad \& \quad l(C) \geqq 2$.

(ix-1) $p \geqq 2, q=0$.

(ix-2) $\quad p \geqq 1, q \geqq 1$.

Considering Property 5, we can reduce (ix-1) and (ix-2) to Property 6 (IV-1) and $(\mathrm{V}-1)$, respectively.

(x) The case $l(A) \geqq 2 \& l(B) \geqq 2 \& l(C)=0$.

Considering Property 5, we can reduce this case to Property 6 (III-2).

(xi) The case $l(A) \geqq 3 \quad \& l(B)=1 \& l(C)=0$.

Considering Property 5, we can reduce this case of Property 6 (I-6).

(xii) The case $l(A) \geqq 5 \quad \& \quad l(B)=0 \& l(C)=0$.

Considering Property 5 , we can reduce this case to Property 6 (I-5).

(xiii) The case $l(A)=1 \& l(B) \geqq 3 \quad \& \quad l(C)=0$.

Considering Property 5, we can reduce this case to Property 6 (I-3).

(xiv) The case $l(A)=0 \& l(B) \geqq 4 \quad \& l(C)=0$.

Considering Property 5, we can reduce this case to Property 6 (III-3).

This completes the proof. 


\section{REFERENCES}

[1] H.S.M. Coxeter AND W.O.J. Moser, Generators and Relations for Discrete Groups, Springer Verlag 1972 (Third edition).

[2] H. KimurA, On automorphism groups of compact Riemann surfaces with prescribed group structure - The cases of $D_{8}$ and $Q_{8}$ - (preprint)

[3] A. Kuribayashi And H. Kimura, Automorphism groups of compact Riemann surfaces of genus five, J. Algebra, vol. 134 (1990), 80-103.

[4] I. KURIBAyAshi, On automorphisms of prime order of a Riemann surface as matrices, Manuscripta Math. 44 (1983), 103-108.

[5] I. KuRibayashi, On an algebraization of the Riemann-Hurwitz relation, Kodai Math. J., 7 (1984), 222-237.

[6] I. KuRIBAyAshi, Classification of automorphism groups of compact Riemann surfaces of genus two. (Preprint at Tsukuba 1986).

[7] I. KuRIBAyAshi, On automorphism groups of a curve as linear groups, J. Math. Soc. Japan, 39 (1987), 51-77.

[Atlas] J. H. Conway, R. T. Curtis, S. P. Norton, R. A. Parker, and R. A. Wilson, Atlas of finite groups, Clarendon PREss, Oxford, 1985.

[G] D. Gorenstein, Finite Groups, Harper \& Row, Publishers, New York, 1968.

Tokyo Institute of Technology

Department of Mathematics

MEGURO-KU, TOKYO, JAPAN

AND

FELlowships of THE JAPAN Society

For the Promotion of Science

FOR JAPANESE JUNior SCIENTISTS 